\title{
SIMULATION OF CONTROL OF MULTI-VARIABLE CONTROL LOOP: STEAM TURBINE
}

\author{
Navrátil, P. \& Balátě, J. \\ Faculty of Applied Informatics, Tomas Bata University in Zlín, Nad Stráněmi 4511, \\ 76005 Zlín, Czech Republic \\ E-Mail: pnavratil@fai.utb.cz; balate@fai.utb.cz
}

\begin{abstract}
The paper deals with the description and simulation verification of one of possible methods to control of multivariable control loops. In this case, the so called main controllers, binding members and correction members are used. The proposed method of control combines classical way to ensure of autonomy of control loop via binding members and the use of the method of single-variable branched control loops with measurement of dominant disturbance variables to ensure of invariance of control loop via correction members. Main controllers can be proposed by arbitrary synthesis method. Simulation verifications of the control method are carried out for three-variable loop of a steam turbine.

(Received in April 2010, accepted in January 2011. This paper was with the authors 1 month for 1 revision.)
\end{abstract}

Key Words: MIMO Control Loop, Synthesis, Autonomous Control, Invariant Control

\section{INTRODUCTION}

At large numbers of controlled systems several variables have to be controlled at the same time. The examples of these systems are e.g. air-conditioning plants, reactors, steam boilers, turbines, distillation columns, etc [1]. In these cases, it means that there is not only larger number of independent SISO (single-variable) control loop. These control loops are complex with several controlled variables where separate variables are not mutually independent. Mutual coupling of controlled variables is usually given by simultaneous action of each of input (manipulated and disturbance) variables of controlled plant to all output (controlled) variables. These control loops are called MIMO (multi-variable) control loops and they consist of mutually influencing simpler control loops [1-2]. Special case of MIMO control loop is SISO control loop having only one input signal (manipulated variable, disturbance variable) and one output signal (controlled variable) [3].

One of above-mentioned examples of multi-variable controlled system is a steam turbine [4-6]. In the experimental part of the paper is considered simulation example of three-variable controlled system of steam turbine [4]. Proposed method to control of the three-variable controlled system uses the so called main controllers, binding members and correction members [1]. The main controllers are designed via classical SISO synthesis methods (Ziegler Nichols step response method, method of desired model) [1, 7-10] and also via polynomial approach (pole placement method) for SISO control loop [11-12]. Binding members and correction members ensure autonomy of control loop and invariance of control loop. All simulation experiments were performed in the simulation mathematical software MATLAB/SIMULINK version 6.5 [13].

\section{MULTI-VARIABLE CONTROL LOOP}

It is considered multi-variable control loop with measurement of disturbance (see Fig. 1) where $\boldsymbol{G}_{\boldsymbol{S}}(s), \boldsymbol{G}_{\boldsymbol{R}}(s), \boldsymbol{G}_{\boldsymbol{S} \boldsymbol{V}}(s)$ and $\boldsymbol{G}_{\boldsymbol{K} C}(\mathrm{~s})$ are transfer matrixes of a controlled plant, controller, 
disturbance variables and correction members. Signal $\boldsymbol{Y}(s)[n \times 1]$ is a vector of controlled variables, $\boldsymbol{W}(s)$ is a vector of setpoints, $\boldsymbol{U}(s)[n \times 1]$ is a vector of manipulated variables, $\boldsymbol{V}(s)$ [ $m \times 1]$ is a vector of disturbance variables and it is considered $m \leq n$ [14].

Transfer matrixes of controlled plant $\boldsymbol{G}_{\boldsymbol{S}}(s)$ and transfer matrix of disturbance variables $\boldsymbol{G}_{\boldsymbol{S V}}(s)$ are considered in forms:

$$
\begin{gathered}
\boldsymbol{G}_{S}(s)=\left[\begin{array}{cccc}
S_{11} & S_{12} & \cdots & S_{1 n} \\
S_{21} & S_{22} & \cdots & S_{2 n} \\
\vdots & \vdots & \cdots & \vdots \\
S_{n 1} & S_{n 2} & \cdots & S_{n n}
\end{array}\right] ; S_{i j}=\frac{Y_{S, i}(s)}{U_{j}(s)} i=1, \ldots, n, j=1, \ldots, n \\
\boldsymbol{G}_{S V}(s)=\left[\begin{array}{cccc}
S_{V 11} & S_{V 12} & \cdots & S_{V 1 m} \\
S_{V 21} & S_{V 22} & \cdots & S_{V 2 m} \\
\vdots & \vdots & \cdots & \vdots \\
S_{V n 1} & S_{V n 2} & \cdots & S_{V n m}
\end{array}\right] ; S_{V i j}=\frac{Y_{S V, i}(s)}{V_{j}(s)} i=1, \ldots, n, j=1, \ldots, m, m \leq n
\end{gathered}
$$

Transfer matrixes of controller $\boldsymbol{G}_{\boldsymbol{R}}(s)$ and transfer matrix of correction member $\boldsymbol{G}_{\boldsymbol{K} \boldsymbol{C}}(s)$ are considered in forms:

$$
\begin{gathered}
\boldsymbol{G}_{R}(s)=\left[\begin{array}{cccc}
R_{11} & R_{12} & \cdots & R_{1 n} \\
R_{21} & R_{22} & \cdots & R_{2 n} \\
\vdots & \vdots & \cdots & \vdots \\
R_{n 1} & R_{n 2} & \cdots & R_{n n}
\end{array}\right] ; R_{i j}=\frac{U_{R, i}(s)}{E_{j}(s)} i=1, \ldots, n, j=1, \ldots, n \\
\boldsymbol{G}_{K C}(s)=\left[\begin{array}{cccc}
K C_{11} & 0 & \cdots & 0 \\
0 & K C_{22} & \cdots & 0 \\
\vdots & \vdots & \cdots & \vdots \\
0 & 0 & \cdots & K C_{n n}
\end{array}\right] ; K C_{i j}=\frac{U_{K C, i}(s)}{V_{j}(s)} \quad i=1, \ldots, n, j=1, \ldots, m, m \leq n
\end{gathered}
$$

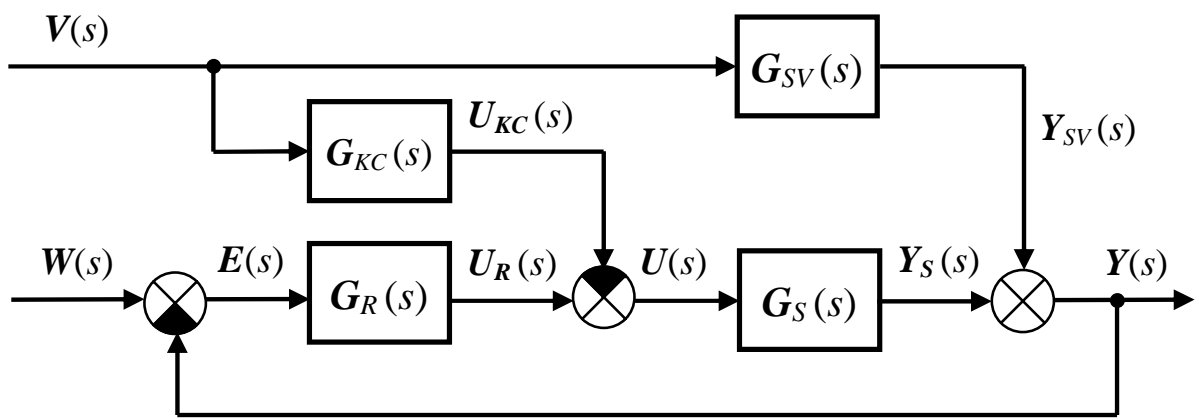

Figure 1: MIMO control loop with measurement of disturbance.

\subsection{Autonomy and invariance of control loop}

Beside stability and quality of control it is often required at synthesis of multi-variable control loop that one control variable causes a change of only one corresponding (proper) controlled variable. Such control loop is called autonomous. Elimination of influence of measurable disturbance variables on controlled variables can also be a further requirement. Such control loop is called invariant. Control loop at which the influence of disturbances on controlled variables is completely eliminated are called absolutely invariant. Control loops at which the influence of disturbance variables is eliminated only partially (e.g. only in steady state) are called approximately invariant. They are often called also invariant up to $\varepsilon$ where $\varepsilon$ is an error caused by incomplete elimination of influence of disturbances [1]. 
In order to ensure autonomy and invariance of control loop we start from a closed loop transfer matrix $\boldsymbol{G}_{W / Y}(s)$ and disturbance transfer matrix $\boldsymbol{G}_{V / Y}(s)$, therefore:

$$
\begin{gathered}
\boldsymbol{G}_{W / Y}(s)=\left[\boldsymbol{I}+\boldsymbol{G}_{S}(s) \boldsymbol{G}_{R}(s)\right]^{-1} \boldsymbol{G}_{S}(s) \boldsymbol{G}_{R}(s) \\
\boldsymbol{G}_{V / Y}(s)=\left[\boldsymbol{I}+\boldsymbol{G}_{S}(s) \boldsymbol{G}_{R}(s)\right]^{-1}\left[\boldsymbol{G}_{S V}(s)-\boldsymbol{G}_{S}(s) \boldsymbol{G}_{K C}(s)\right]
\end{gathered}
$$

\section{Autonomy of control loop}

It results from (5) that the control loop is autonomous when the matrix $\boldsymbol{G}_{S}(s) \boldsymbol{G}_{R}(s)$ is diagonal. On the basis of this condition it is possible to derive the following relation:

$$
\frac{R_{k l}}{R_{m l}}=\frac{s_{l k}}{s_{l m}} \quad k, l, m=<1, \ldots, n>, s_{l m} \neq 0
$$

where $s_{l k}, s_{l m}$ are algebraic supplements of separate elements of a transfer matrix of controlled plant $\boldsymbol{G}_{\boldsymbol{S}}(\mathrm{s})$ (1) and $R_{k l}, R_{m l}$ are separate members of a transfer matrix of controller $\boldsymbol{G}_{\boldsymbol{R}}(s)$ (binding members) (3).

Main (diagonal) controllers $R_{11}, R_{22}, R_{33}$ etc. are usually known already from the first design of conception of control. The relation (7) is therefore used for calculation of all remaining members of matrix controller i.e. for calculation of transfers of binding members, which are aside-from diagonal elements of transfer matrix of controller $\boldsymbol{G}_{R}(s)$.

\section{Invariance of control loop}

For ensuring absolute invariance it is necessary that the disturbance transfer matrix $\boldsymbol{G}_{V / Y}(s)$ (6) is zero. This is possible if the following relation is valid:

$$
\boldsymbol{G}_{K C}(s)=\boldsymbol{G}_{S}^{-1}(s) \boldsymbol{G}_{S V}(s)
$$

At design of correction members $K C$, the task of which is to eliminate the influence of disturbance variable on control loop, internal couplings are omitted at MIMO control loop and thus $n$ SISO branched control loops with measuring of a disturbance variable are gained. Connection of all SISO control loops is the same and they differ only in separate transfers of controlled plants, controllers, correction members and disturbance variables [1]. Common connection of these control loops is presented in the following figure.

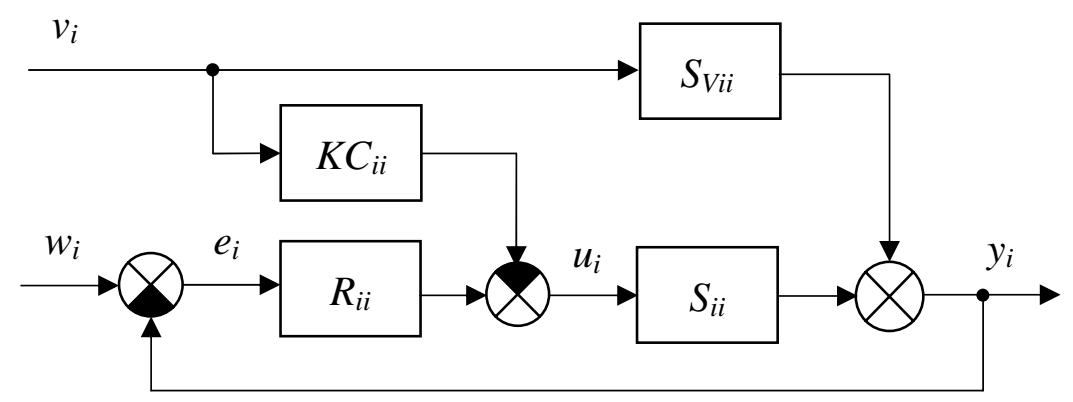

Figure 2: Block diagram of SISO control loop with measuring of disturbance variable $v_{i}$.

Correction members $K C$ are determined from (8) i.e. from the condition of invariance. The invariance of the control loop is ensured, according to the above mentioned method, by using analogy of SISO branched control loops with measuring of disturbance variable $v_{i}$. Transfer functions of correction members $K C$ are gained by using (9) in the following form:

$$
K C_{i i}=\frac{S_{V i i}}{S_{i i}} \quad i=<1, \ldots, n>, S_{i i} \neq 0
$$


where $S_{V i i}$ are separate members of transfer matrix of disturbance variables $\boldsymbol{G}_{\boldsymbol{S V}}(\mathrm{s})$ and $S_{i i}$ are separate members of transfer matrix of controlled plant $\boldsymbol{G}_{\boldsymbol{S}}(s)$.

\subsection{Synthesis of multi-variable control loop}

In practice the possible approximate solution of MIMO control loop is applied from analysis of MIMO control loop and really used control schemes in particular technological equipments $[1,11]$. One of the possible methods of solution of MIMO control loops synthesis is described in the following part of this paper. Generally it is possible to divide this solution into three parts:

- Design of main controllers (diagonal controllers) by arbitrary synthesis method of SISO control loops, i.e. design of parameters of main controllers for $n$ SISO control loops $\left(R_{11}, R_{22}, \ldots, R_{n n}\right)$. Here, it is considered that original diagonal transfer functions $S_{i i}(i=1, \ldots, n)$ of transfer matrix of controlled plant $\boldsymbol{G}_{S}(s)$ are modified to diagonal transfer functions $S_{i i, x}(i=1, \ldots, n)$. In these modified transfer functions influences of aside-from diagonal transfer functions of transfer matrix of controlled plant $\boldsymbol{G}_{S}($ s), i.e. $S_{i j}(i \neq j, i, j=1, \ldots, n)$ on original diagonal transfer functions i.e. $S_{i i}(i=1, \ldots, n)$ are included. Transfer functions $S_{i i, x}$, i.e $S_{11, x}, S_{22, x}, S_{33, x}$ etc. are determined from (10) by using (5) and (7):

$$
S_{i i, x}=\sum_{j=1}^{n} S_{i j} \frac{s_{i j}}{S_{i i}} \quad i=<1, \ldots, n>, s_{i i} \neq 0
$$

where $s_{i i}, s_{i j}$ are algebraic supplements of separate elements of a transfer matrix of controlled plant $\boldsymbol{G}_{\boldsymbol{S}}(s)$ and $S_{i j}$ are separate members of a transfer matrix of controlled plant $\boldsymbol{G}_{\boldsymbol{S}}(s)$.

- Assuring of autonomy of control loop via binding members (7) of transfer matrix of controller $\boldsymbol{G}_{R}(s)$.

- Assuring of invariance control loop via correction members $K C$ (9) by using $n$ SISO branched control loops with measuring of disturbance variables.

\section{SIMULATION EXAMPLE}

\subsection{Three-variable controlled plant of steam turbine}

Steam turbine is a typical example of MIMO controlled plant. In this case it is considered the turbine with two controlled withdrawals which drives electric generator supplying determined part of electric network (it means the turbine operates without phasing into power network). The scheme of three-variable controlled plant of steam turbine is shown in Fig. 3.

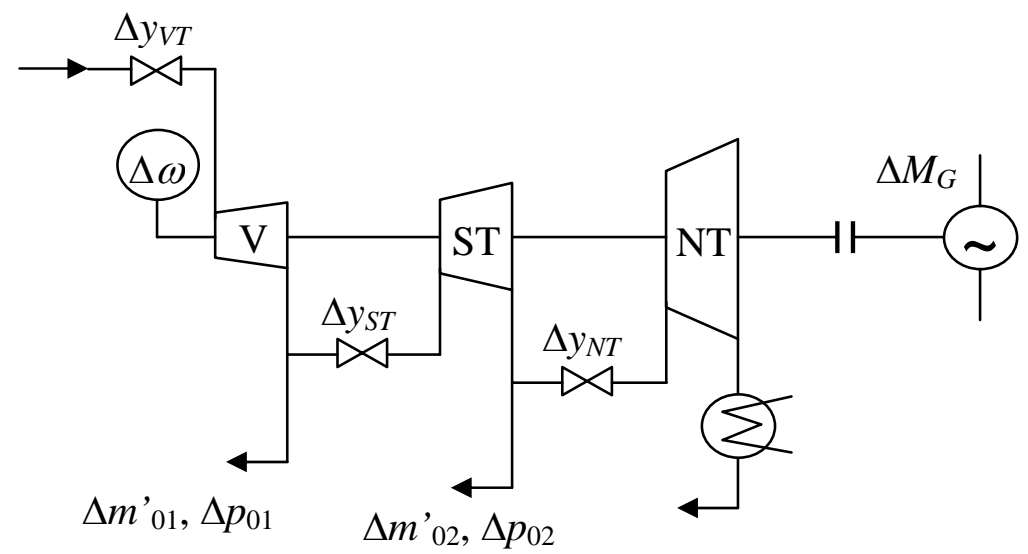

Figure 3: Three-variable controlled plant of steam turbine. 
Denominations in Fig. 3 mean: $\Delta M_{G}$ - change of electric load of turbo-generator, $\Delta m^{\prime}{ }_{01}$, $\Delta m^{\prime}{ }_{02}$ - change of mass flow of withdrawn steam, $\Delta \omega$ - change of angular speed of turbogenerator, $\Delta p_{01}, \Delta p_{02}$ - change of steam pressure in corresponding withdrawals, $\Delta y_{V T}, \Delta y_{S T}$, $\Delta y_{N T}$ - change of opening position of control valves of high-pressure, medium-pressure and low-pressure part of turbine.

Controlled variables are $\Delta \omega, \Delta p_{01}, \Delta p_{02}$, manipulated variables are $\Delta y_{V T}, \Delta y_{S T}, \Delta y_{N T}$ and disturbance variables are $\Delta M_{G}, \Delta m^{\prime}{ }_{01}, \Delta m^{\prime}{ }_{02}$.

\subsection{Mathematical model of steam turbine}

Resulting differential equations for creating mathematical model of the controlled plant were gained already after deriving and using linearization from the project OTROKOVICE elaborated by the firm ALSTOM Power [4]. The mathematical model consists of three equations, i.e. equation of moment balance (11) and equations of flow through flow spaces (12), (13).

$$
\begin{gathered}
518.4 \Delta \dot{\omega}=-63.3 \Delta \omega+656.9 \Delta p_{01}+4611.7 \Delta p_{02}+1007.3 \Delta y_{V T}+200.6 \Delta y_{S T}+ \\
+121.5 \Delta y_{N T}-\Delta M_{G} \\
1.865 \Delta \dot{p}_{01}=-1.610 \Delta p_{01}+0.167 \Delta p_{02}+1.523 \Delta y_{V T}-0.361 \Delta y_{S T}-\Delta m_{01}^{\prime} \\
13.45 \Delta \dot{p}_{02}=1.563 \Delta p_{01}-10.517 \Delta p_{02}+0.361 \Delta y_{S T}-0.222 \Delta y_{N T}-\Delta m_{02}^{\prime}
\end{gathered}
$$

It is possible to re-write the above differential equations (11), (12) and (13) into betterarranged form by introducing relative values, with regard to starting stable state-operational, i.e. to calculated point, at which relation of values can be generally written in the form $\varphi_{X}=\Delta X /(X)_{0}$

where:

$$
\begin{array}{lll}
(\omega)_{0}=628.3[\mathrm{rad} / \mathrm{s}] & \left(p_{01}\right)_{0}=14[\mathrm{bar}] & \left(p_{02}\right)_{0}=1,55[\mathrm{bar}] \\
\left(y_{V T}\right)_{0}=19.15[\mathrm{~mm}] & \left(y_{S T}\right)_{0}=59.9[\mathrm{~mm}] & \left(y_{N T}\right)_{0}=69.8[\mathrm{~mm}] \\
\left(M_{G}\right)_{0}=39789[\mathrm{Nm}] & \left(m_{01}^{\prime}\right)_{0}=6.94[\mathrm{~kg} / \mathrm{s}] & \left(m_{02}^{\prime}\right)_{0}=6.94[\mathrm{~kg} / \mathrm{s}]
\end{array}
$$

hence:

$$
\begin{gathered}
325710.7 \dot{\varphi}_{\omega}=-39771.4 \varphi_{\omega}+9196.6 \varphi_{p_{01}}+7148.1 \varphi_{p_{02}}+19289.8 \varphi_{y_{V T}} \\
+12015.9 \varphi_{y_{S T}}+8480.7 \varphi_{y_{N T}}-39789 \varphi_{M_{G}} \\
26.110 \dot{\varphi}_{p_{01}}=-22.540 \varphi_{p_{01}}+0.259 \varphi_{p_{02}}+29.165 \varphi_{y_{V T}}-21.624 \varphi_{y_{S T}}-6.94 \varphi_{m_{01}^{\prime}} \\
20.848 \dot{\varphi}_{p_{02}}=21.882 \varphi_{p_{01}}-16.301 \varphi_{p_{02}}+21.624 \varphi_{y_{S T}}-15.496 \varphi_{y_{N T}}-6.94 \varphi_{m_{02}^{\prime}}
\end{gathered}
$$

Three algebraic equations are gained from differential equations (15) - (17), by using the sentence about differentiation of the original, out of which after arrangement it is possible to put together transfer matrix of the controlled plant $\boldsymbol{G}_{S}(s)(20)$ and disturbance variables $\boldsymbol{G}_{S V}(s)(21)$. The Laplace transform of an output (controlled) variable is given by the following relation:

$$
\boldsymbol{Y}(s)=\boldsymbol{G}_{S}(s) \boldsymbol{U}(s)+\boldsymbol{G}_{S V}(s) \boldsymbol{V}(s)
$$

After substitution we receive:

$$
\left[\begin{array}{c}
\varphi_{\omega} \\
\varphi_{p_{01}} \\
\varphi_{p_{02}}
\end{array}\right]=\boldsymbol{G}_{S}(s)\left[\begin{array}{c}
\varphi_{y_{V T}} \\
\varphi_{y_{S T}} \\
\varphi_{y_{N T}}
\end{array}\right]+\boldsymbol{G}_{S V}(s)\left[\begin{array}{c}
\varphi_{M_{G}} \\
\varphi_{m_{01}^{\prime}} \\
\varphi_{m_{02}^{\prime}}
\end{array}\right]
$$


where:

$$
\begin{gathered}
\boldsymbol{G}_{S}(s)=\left[\begin{array}{ccc}
\frac{0.73 s^{2}+1.59 s+1.106}{12.32 s^{3}+21.78 s^{2}+10.67 s+1} & \frac{0.455 s^{2}+0.740 s+0.087}{12.32 s^{3}+21.78 s^{2}+10.67 s+1} & \frac{0.321 s^{2}+0.327 s+0.037}{12.32 s^{3}+21.78 s^{2}+10.67 s+1} \\
\frac{1.681 s+1.314}{1.505 s^{2}+2.475 s+1} & \frac{-1.246 s-0.959}{1.505 s^{2}+2.475 s+1} & \frac{-0.011}{1.505 s^{2}+2.475 s+1} \\
\frac{1.764}{1.505 s^{2}+2.475 s+1} & \frac{1.561 s+0.039}{1.505 s^{2}+2.475 s+1} & \frac{-1.118 s-0.966}{1.505 s^{2}+2.475 s+1}
\end{array}\right] \\
\boldsymbol{G}_{S V}(s)=\left[\begin{array}{cccc}
\frac{-1.505 s^{2}-2.477 s-1}{12.32 s^{3}+21.78 s^{2}+10.67 s+1} & \frac{-0.092 s-0.148}{12.32 s^{3}+21.78 s^{2}+10.67 s+1} & \frac{-0.090 s-0.079}{12.32 s^{3}+21.78 s^{2}+10.67 s+1} \\
0 & \frac{-0.400 s-0.313}{1.505 s^{2}+2.475 s+1} & \frac{-0.005}{1.505 s^{2}+2.475 s+1} \\
0 & \frac{-0.420}{1.505 s^{2}+2.475 s+1} & \frac{-0.501 s-0.432}{1.505 s^{2}+2.475 s+1}
\end{array}\right]
\end{gathered}
$$

Step response of transfer matrix of the controlled plant $\boldsymbol{G}_{S}(s)$ and of transfer matrix of disturbance variables $\boldsymbol{G}_{S V}(s)$ are presented in the following figures (see Figs. 4 and 5) [13].

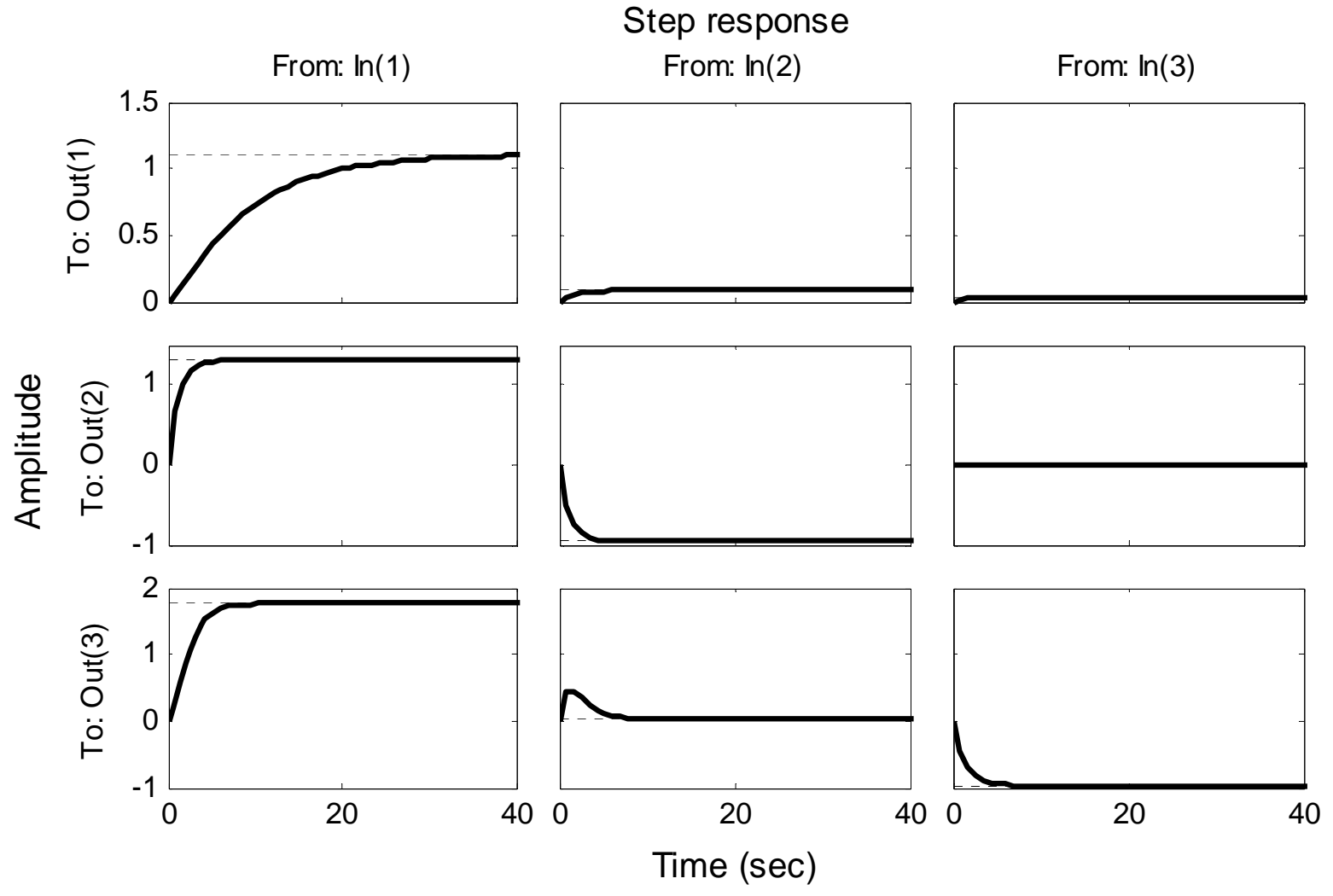

Figure 4: Step response for transfer matrix of the controlled plant $\boldsymbol{G}_{S}(s)$.

\subsection{Synthesis of three-variable control loop of a steam turbine}

The principal described in the section 2.2 is used at solution of synthesis of the three-variable control loop. First transfers function of main controllers $R_{11}, R_{22}, R_{33}$ are determined for modified diagonal transfer functions $S_{11, x}, S_{22, x}$ and $S_{33, x}$ by using (10) or (22) then autonomy of control loop by using (7) is being solved and in the end fulfilment of the condition of invariance (approximate invariance) of control loop is ensured by using (9). 


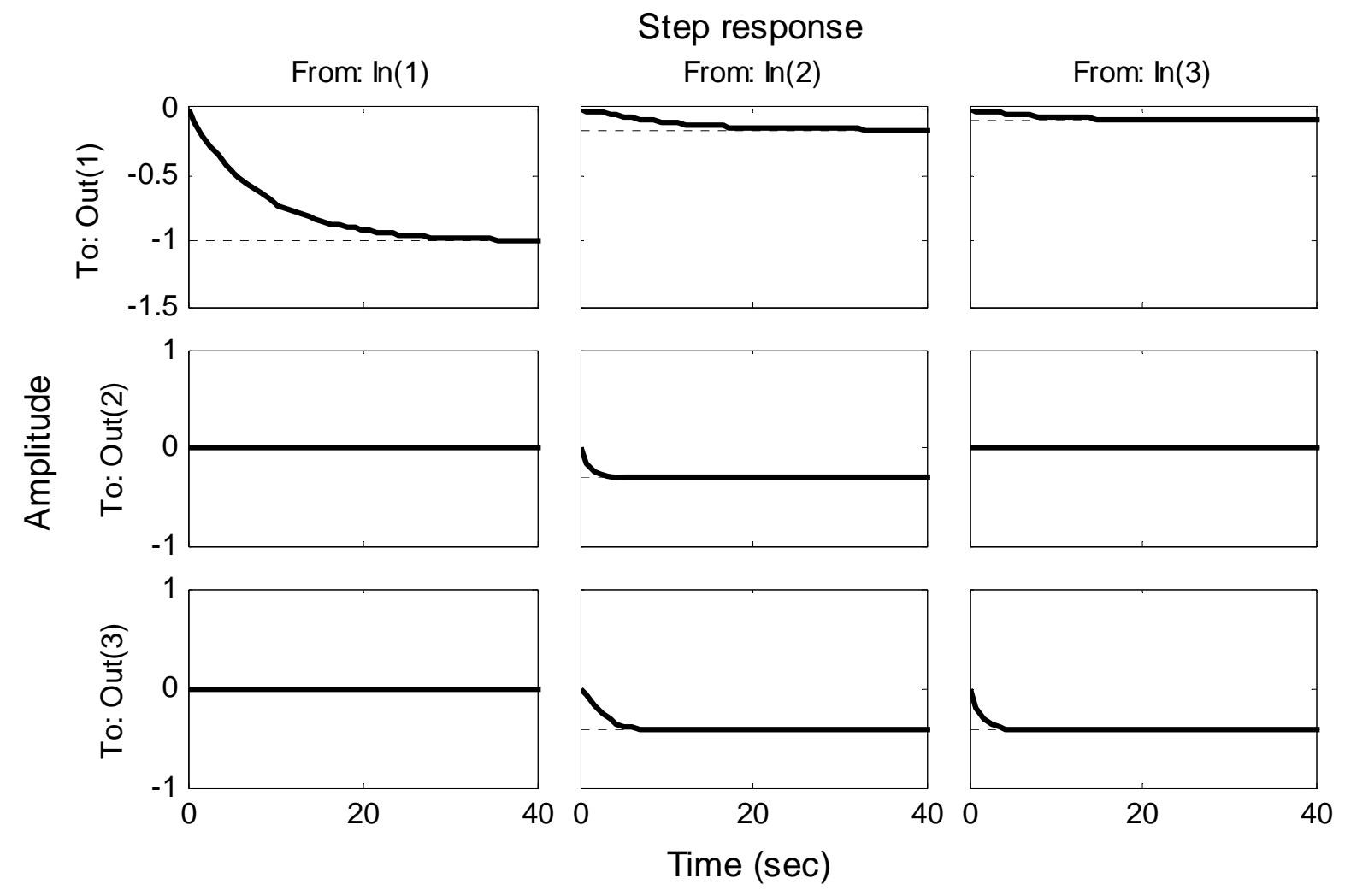

Figure 5: Step response for transfer matrix of disturbance variables $\boldsymbol{G}_{S V}(s)$.

Eq. (10) was used to calculation of transfer functions $S_{i i, x}$, hence:

$$
\begin{aligned}
& S_{11, x}(s)=S_{11} \frac{s_{11}}{s_{11}}+S_{12} \frac{s_{12}}{s_{11}}+S_{13} \frac{s_{13}}{S_{11}}=S_{11}+S_{12} \frac{S_{23} S_{31}-S_{21} S_{33}}{S_{22} S_{33}-S_{23} S_{32}}+S_{13} \frac{S_{21} S_{32}-S_{22} S_{31}}{S_{22} S_{33}-S_{23} S_{32}} \\
& S_{22, x}(s)=S_{21} \frac{s_{21}}{S_{22}}+S_{22} \frac{s_{22}}{s_{22}}+S_{23} \frac{s_{23}}{S_{22}}=S_{21} \frac{S_{13} S_{32}-S_{12} S_{33}}{S_{11} S_{33}-S_{13} S_{31}}+S_{22}+S_{23} \frac{S_{12} S_{31}-S_{11} S_{32}}{S_{11} S_{33}-S_{13} S_{31}} \\
& S_{33, x}(s)=S_{31} \frac{S_{31}}{S_{33}}+S_{32} \frac{S_{32}}{S_{33}}+S_{33} \frac{S_{33}}{S_{33}}=S_{31} \frac{S_{12} S_{23}-S_{13} S_{22}}{S_{11} S_{22}-S_{12} S_{21}}+S_{32} \frac{S_{13} S_{21}-S_{11} S_{33}}{S_{11} S_{22}-S_{12} S_{21}}+S_{33}
\end{aligned}
$$

then:

$$
S_{11, x}=\frac{1.294}{8.19 s+1} \quad S_{22, x}=\frac{-1.057}{0.479 s+1} \quad S_{33, x}=\frac{-1.020}{0.964 s+1}
$$

The following methods were used at design of parameters of main controllers (diagonal elements of transfer matrix of controller $\boldsymbol{G}_{R}(s)$ ):

a) Ziegler Nichols step response method $[1,7]$,

b) method of desired model (method of dynamics inversion) [8-10],

c) polynomial method of synthesis for 1DOF (one degree of freedom) configuration (pole placement method) [11-12].

Beside above mentioned methods of design of parameters of main controllers (diagonal elements of transfer matrix of controller $\boldsymbol{G}_{R}(s)$ ) is possible to use also other SISO synthesis methods, e.g. the method of optimal module, the Naslin's method, the symmetrical optimum method, etc. [1, 7, 9]. 
Eq. (7) was used to calculation of binding members $R_{i j}$ (aside-from diagonal elements of transfer matrix of controller $\boldsymbol{G}_{R}(s)$ ). Then these members were determined from following relations:

$$
\begin{array}{ll}
R_{12}(s)=\frac{s_{21}}{s_{22}} R_{22}=\frac{S_{13} S_{32}-S_{12} S_{33}}{S_{11} S_{33}-S_{13} S_{31}} R_{22} & R_{13}(s)=\frac{s_{31}}{s_{33}} R_{33}=\frac{S_{12} S_{23}-S_{13} S_{22}}{S_{11} S_{22}-S_{12} S_{21}} R_{33} \\
R_{21}(s)=\frac{s_{12}}{s_{11}} R_{11}=\frac{S_{23} S_{31}-S_{21} S_{33}}{S_{22} S_{33}-S_{23} S_{32}} R_{11} & R_{23}(s)=\frac{s_{32}}{s_{33}} R_{33}=\frac{S_{13} S_{21}-S_{11} S_{33}}{S_{11} S_{22}-S_{12} S_{21}} R_{33} \\
R_{31}(s)=\frac{s_{13}}{s_{11}} R_{11}=\frac{S_{21} S_{32}-S_{22} S_{31}}{S_{22} S_{33}-S_{23} S_{32}} R_{11} & R_{32}(s)=\frac{s_{23}}{s_{22}} R_{22}=\frac{S_{12} S_{31}-S_{11} S_{32}}{S_{11} S_{33}-S_{13} S_{31}} R_{22}
\end{array}
$$

To determine of correction members $K C_{i i}$ was used relation (9), hence:

$$
K C_{11}=\frac{S_{V 11}}{S_{11}}, K C_{22}=\frac{S_{V 22}}{S_{22}}, K C_{33}=\frac{S_{V 33}}{S_{33}}
$$

Transfer matrix of controllers $\boldsymbol{G}_{R}(s)$ with utilization of chosen methods of synthesis was following:

a) Ziegler Nichols step response method:

$$
\boldsymbol{G}_{R}(s)=\left[\begin{array}{ccc}
\frac{5.731 s+1.656}{s} & \frac{0.507 s^{2}+0.211 s+0.0188}{s(s+2.089)} & \frac{0.201 s^{2}+0.084 s+0.0076}{s(s+1.057)} \\
\frac{7.73 s+2.223}{s} & \frac{-0.410 s-0.118}{s} & \frac{0.271 s^{2}+0.124 s+0.013}{s(s+1.057)} \\
\frac{10.79 s+3.116}{s} & \frac{-0.527 s^{2}-0.082 s+0.024}{s(s+2.089)} & \frac{-0.841 s-0.243}{s}
\end{array}\right]
$$

b) method of desired model:

$$
\boldsymbol{G}_{R}(s)=\left[\begin{array}{cccc}
\frac{0.904 s+0.110}{s} & \frac{0.080 s+0.010}{s} & \frac{0.032 s+0.0041}{s} \\
\frac{1.220 s+0.149}{s} & \frac{-0.065 s-0.135}{s} & \frac{0.043 s+0.0073}{s} \\
\frac{1.702 s+0.208}{s} & \frac{-0.090 s+0.0132}{s} & \frac{-0.133 s-0.140}{s}
\end{array}\right]
$$

c) polynomial method of synthesis for 1DOF configuration (pole placement method):

$$
\boldsymbol{G}_{R}(s)=\left[\begin{array}{ccc}
\frac{0.810 s+0.095}{s} & \frac{0.286 s^{2}+0.960 s+0.118}{s(s+2.089)} & \frac{0.054 s^{2}+0.100 s+0.012}{s(s+1.057)} \\
\frac{1.092 s+0.128}{s} & \frac{-0.231 s-0.747}{s} & \frac{0.073 s^{2}+0.138 s+0.021}{s(s+1.057)} \\
\frac{1.524 s+0.179}{s} & \frac{-0.323 s^{2}-0.995 s+0.153}{s(s+2.089)} & \frac{-0.226 s-0.400}{s}
\end{array}\right]
$$

Transfer matrix of correction members was given by the following relation (it was the same for all three methods): 


$$
\boldsymbol{G}_{K C}(s)=\left[\begin{array}{ccc}
\frac{-2.063 s^{2}-3.394 s-1.371}{s^{2}+2.178 s+1.516} & 0 & 0 \\
0 & \frac{0.321 s+0.251}{s+0.769} & 0 \\
0 & 0 & 0.448
\end{array}\right]
$$

Simulating scheme presented in Fig. 6 is utilized in program environment MATLAB/ SIMULINK [13] for simulating verification of used procedure.

Simulation courses of three-variable control loop of a steam turbine with utilization of chosen SISO synthesis methods, which are used at design of parameters of main controllers, are presented in the following figures (see Figs. 7, 8 and 9). These parameters were chosen at all simulations:

- vector of time for setpoints $\left(t_{w 1}, t_{w 2}, t_{w 3}\right): \quad\left[\begin{array}{lll}50 & 200 & 350\end{array}\right]$

- vector of setpoints $\left(w_{1}, w_{2}, w_{3}\right)$ : $\quad\left[\begin{array}{lll}0.4 & 0.4 & 0.4\end{array}\right]$

- vector of time for disturbances $\left(t_{v 1}, t_{v 2}, t_{v 3}\right)$ : [ [125 275425$]$

- vector of disturbances $\left(v_{1}, v_{2}, v_{3}\right)$ : $\quad\left[\begin{array}{lll}0.2 & 0.2 & 0.2\end{array}\right]$

- total time of simulation $\left(t_{S}\right)$ : $\quad 500$

- time step $(k)$ : 0.05

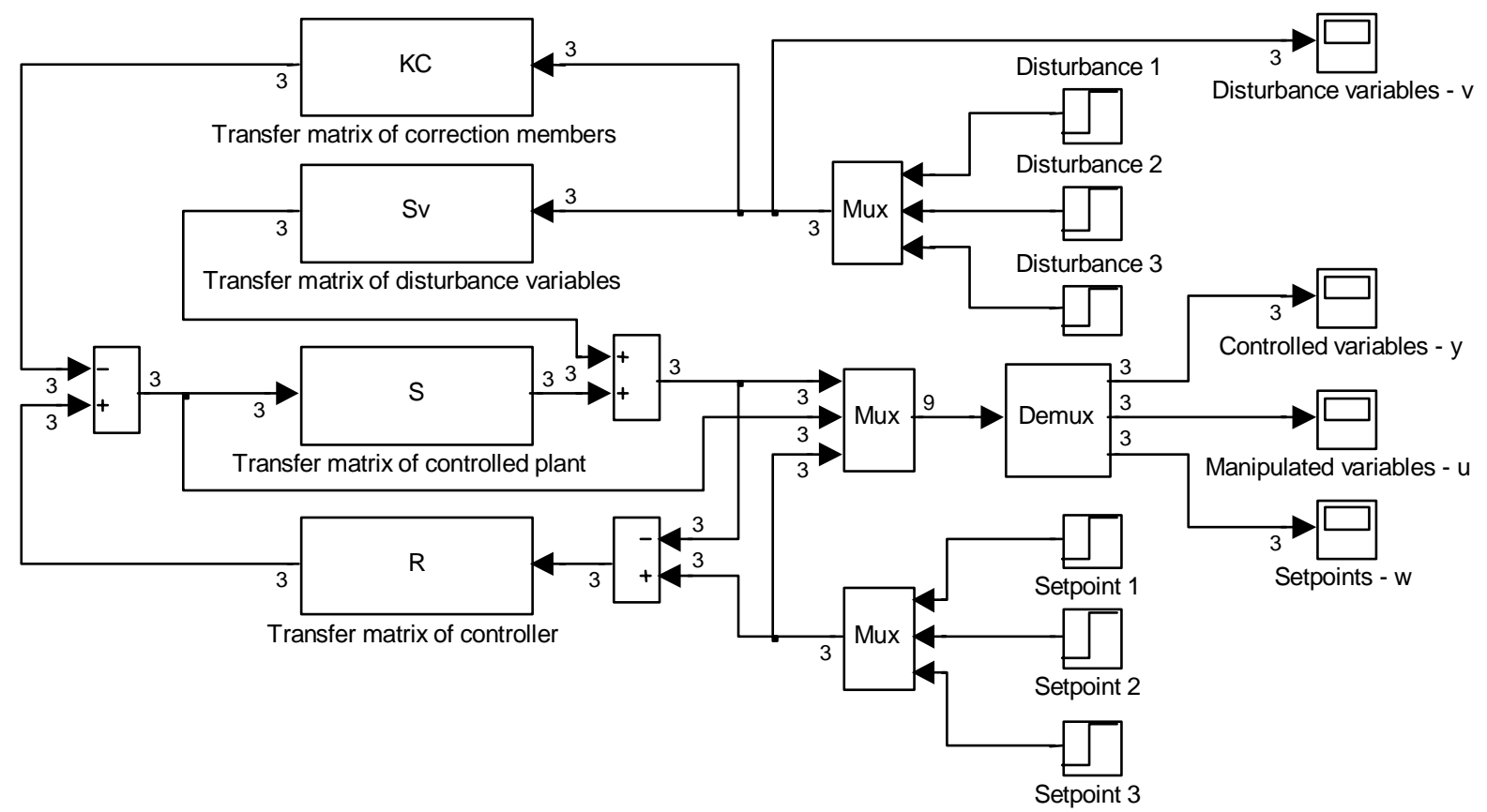

Figure 6: Simulation scheme of control loop.

Variables in the following figures (see Figs. 7, 8 and 9) correspond to variables mentioned in the three-variable control loop of steam turbine (see Fig. 3), i.e.

- controlled variable: $\quad y_{1} \rightarrow \varphi_{\omega}, y_{2} \rightarrow \varphi_{p_{01}}, y_{3} \rightarrow \varphi_{p_{02}}$

- manipulated variable: $\quad u_{1} \rightarrow \varphi_{y_{V T}}, u_{2} \rightarrow \varphi_{y_{S T}}, u_{3} \rightarrow \varphi_{y_{N T}}$

- setpoints (desired value): $w_{1} \rightarrow \varphi_{\omega}, w_{2} \rightarrow \varphi_{p_{01}}, w_{3} \rightarrow \varphi_{p_{02}}$

- disturbance variable: $\quad v_{1} \rightarrow \varphi_{M_{G}}, v_{2} \rightarrow \varphi_{m_{01}^{\prime}}, v_{3} \rightarrow \varphi_{m_{02}^{\prime}}$ 

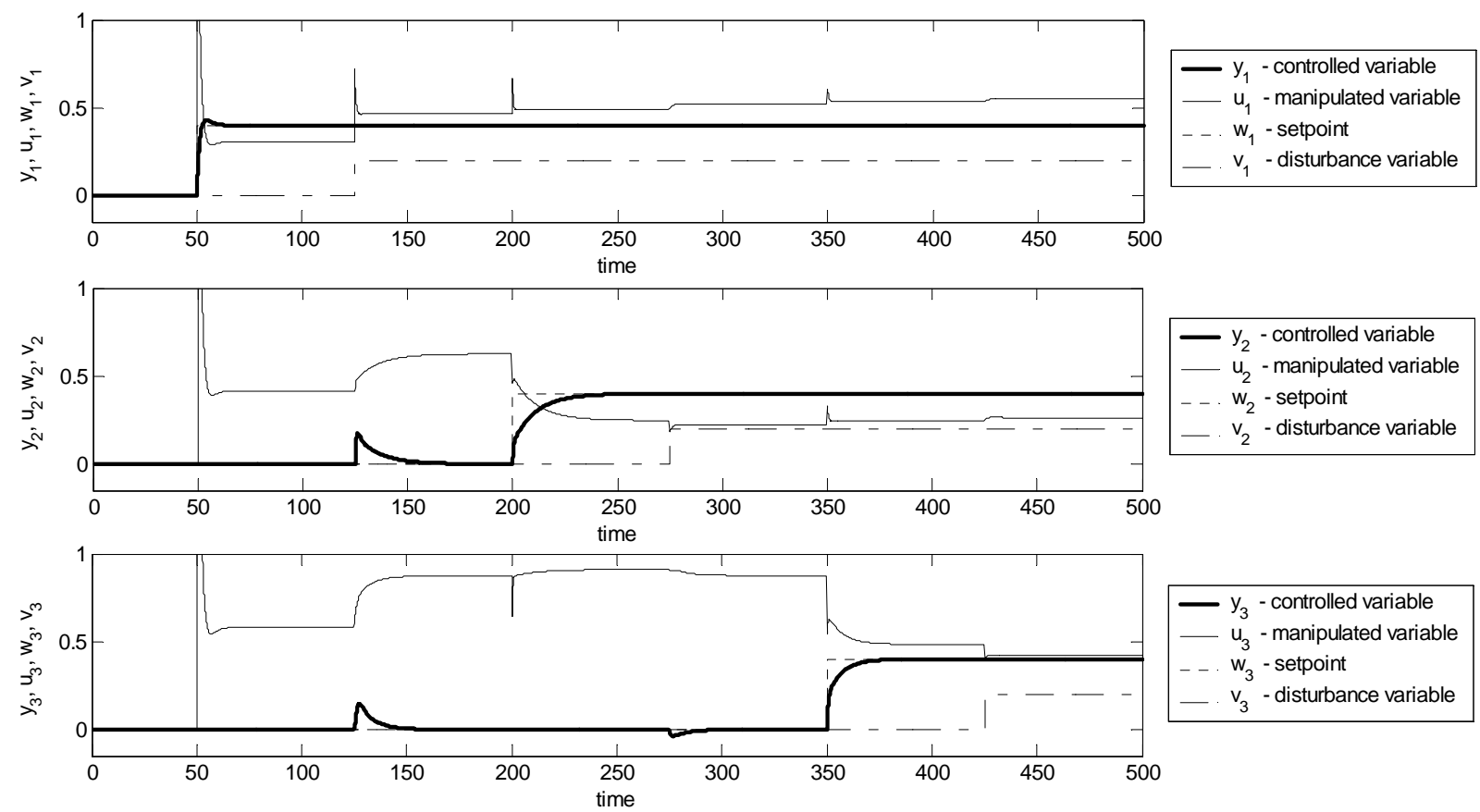

Figure 7: Simulation of control loop with utilization of Ziegler Nichols step response method.
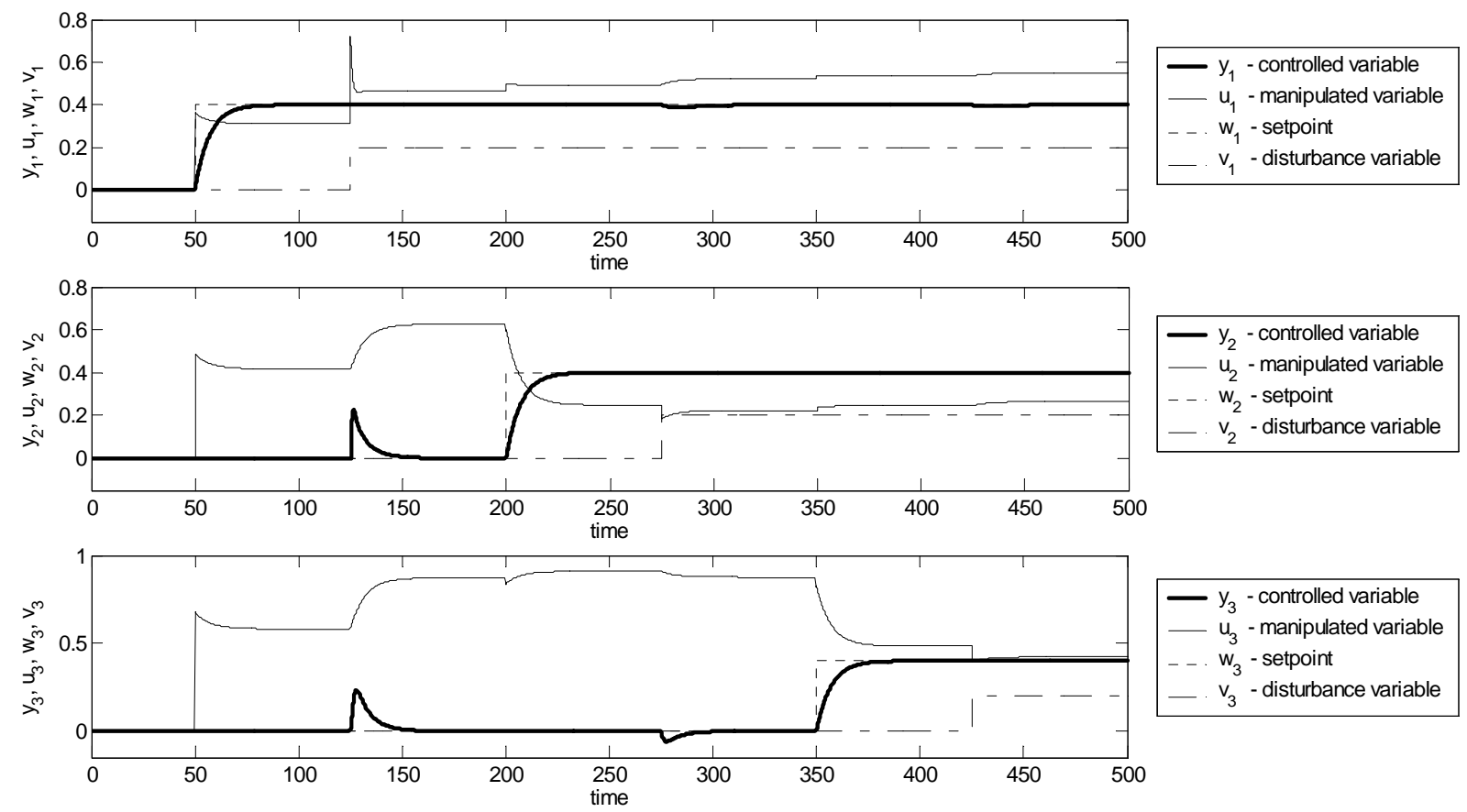

Figure 8: Simulation of control loop with utilization of method of desired model.

\subsection{Evaluation of simulation experiments}

The ISE criterion (30) and ITAE criterion (31) were used for comparison of used methods of synthesis (see Table I), i.e.:

$$
J_{K}=\mathrm{ISE}=\int_{0}^{\infty} e^{2}(t) d t=\int_{0}^{\infty}[w(t)-y(t)]^{2} d t \approx \int_{0}^{t_{r}} e^{2}(t) d t \approx \int_{0}^{t_{s}} e^{2}(t) d t
$$



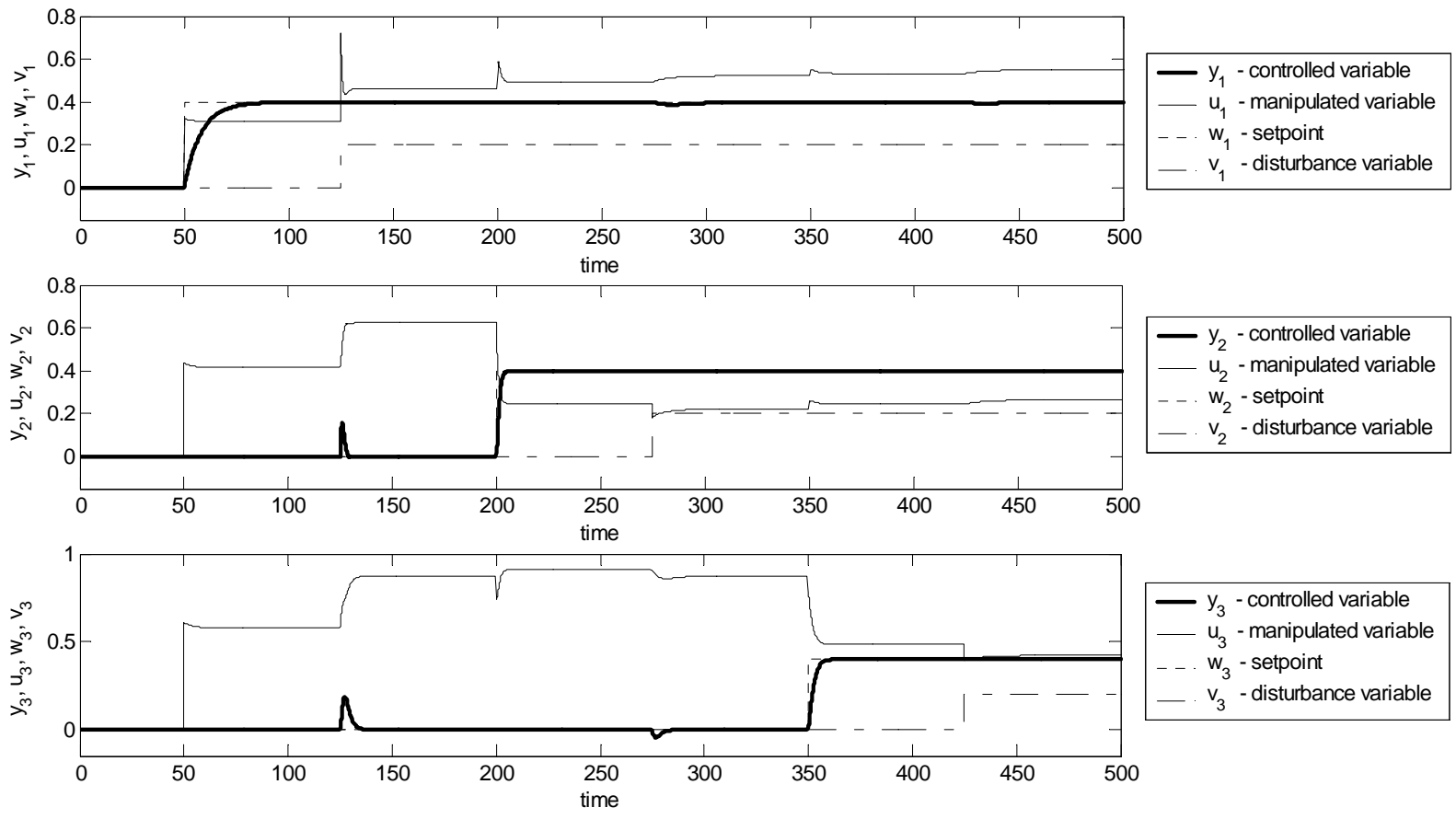

Figure 9: Simulation of control loop with utilization of polynomial method of synthesis.

$$
J_{K}=I T A E=\int_{0}^{\infty} t \cdot|e(t)| d t=\int_{0}^{\infty} t \cdot|(w(t)-y(t))| d t \approx \int_{0}^{t_{r}} t \cdot|e(t)| d t \approx \int_{0}^{t_{s}} t \cdot|e(t)| d t
$$

where $t_{r}$ is time of control, $t_{s}$ is time of simulation, $w(t)$ is setpoint, $y(t)$ is controlled variable, $e(t)$ is control error (see Fig. 10).

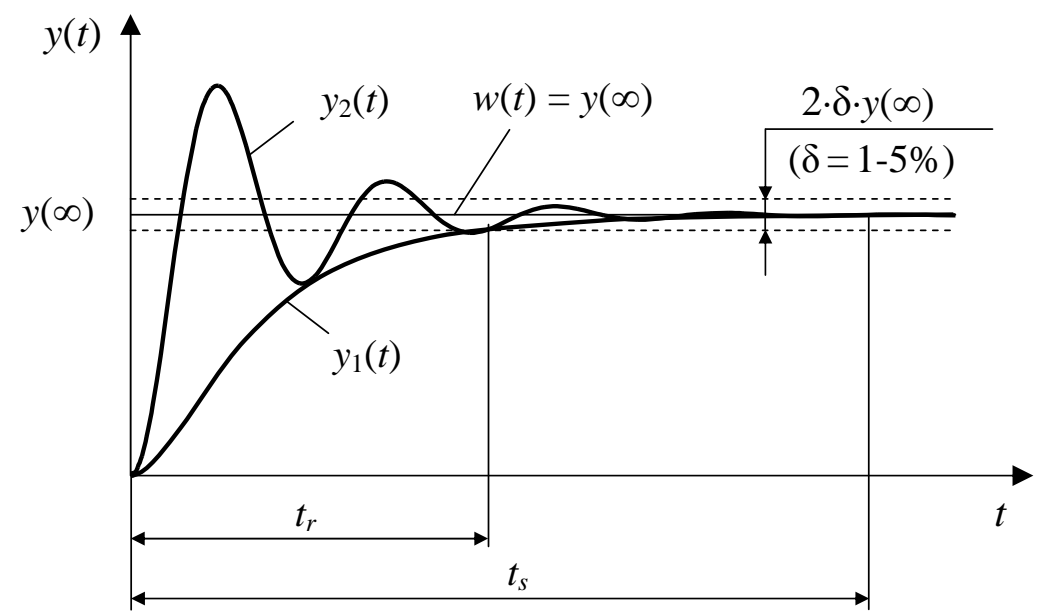

Figure 10: The possible courses of control process.

Optimal adjustment of control loop is considered here from the point of view of minimal size of ISE criterion or ITAE criterion. However quite different point of view can be rally considered for optimal adjustment. Namely requirements for the shortest time of control and for the smallest overshooting are generally valid for optimal adjustment. However these requirements are antagonistic and therefore the optimal adjustment of controller is always a compromise between them. 
Table I: Quality of control for three-variable control loop.

\begin{tabular}{|c|c|c|c|c|c|c|}
\hline \multirow{2}{*}{ Method of synthesis *) } & \multicolumn{2}{|c|}{$\boldsymbol{J}_{\boldsymbol{K} 1}$} & \multicolumn{2}{c|}{$\boldsymbol{J}_{\boldsymbol{K} 2}$} & \multicolumn{2}{c|}{$\boldsymbol{J}_{\boldsymbol{K} 3}$} \\
\cline { 2 - 7 } & $\boldsymbol{I S E}$ & ITAE & ISE & ITAE & ISE & ITAE \\
\hline 1 & 0.0860 & 35.36 & 0.6418 & 933.7 & 0.3355 & 850.1 \\
\hline 2 & 0.5666 & 290.8 & 0.7634 & 801.1 & 0.8978 & 1473.0 \\
\hline 3 & 0.6453 & 343.5 & 0.1518 & 143.6 & 0.3373 & 522.0 \\
\hline
\end{tabular}

*) Numbers in the table in the column "Method of synthesis" designate the used method of synthesis at design of main (diagonal) controllers, i.e.:

1 - Ziegler Nichols step response method,

2 - method of desired model,

3 - polynomial method of synthesis (pole placement method).

It is obvious from the simulation of control loop shown above (see Figs. 7, 8 and 9) that the condition of autonomy was fulfilled. Fulfilment of autonomy condition was ensured by means of using binding members $R_{i j}$ (aside-from-diagonal elements of transfer matrix of controller $\boldsymbol{G}_{R}(s)$ ). Binding members were determined from so called main controllers, which are main diagonal elements of the transfer matrix of controller. The determination of main controllers is carried out by any SISO synthesis method for modified diagonal elements of the transfer matrix of controlled plant. From the simulation of control loop is also obvious that the control loop is invariant or let us say approximately invariant, i.e. that influence of disturbance variables is eliminated only at steady state. Fulfilment of the condition of invariance was ensured by means of correction members $K C_{i i}$ which are considered for elimination of influence of dominant disturbance variables by means of using analogy of SISO branched control loop with measurement of disturbance $v$. Correction members were determined from transfer matrix of the controlled plant and disturbance variables.

\section{CONCLUSION}

The main goal of this paper was to describe and show one of the possible ways to control of MIMO control loop. Advantage of proposed and used method of control is its simplicity.

In this case already known SISO synthesis method can be used to design of main controllers. The designed method combines classical way to ensure of autonomy of control loop via binding members and the use of the method of SISO branched control loops with measurement of dominant disturbance variables to ensure of invariance of control loop by means of correction members. Simulation verification of proposed method of control was presented on three-variable control loop of steam turbine.

The proposed control method is valid under the following conditions, i.e. this method can be used only for MIMO controlled system with same number input and output signals and also the method does not ensure absolute invariance of control loop. Designed parameters of matrix controllers and correction members have good results of the control and fulfilled basic control requirements such as the stability, the reference signal tracking and disturbance attenuation.

The future work will be focused on the elimination of limitations of proposed method and also simulation verification of proposed method for other MIMO controlled systems.

\section{ACKNOWLEDGEMENTS}

This work was supported by the Ministry of Education of the Czech Republic under grant No. MSM 7088352102. 


\section{REFERENCES}

[1] Balate, J. (2004). Automatic Control, $2^{\text {nd }}$ edition, BEN - technical literature, Praha (in Czech)

[2] Goodwin, G. C.; Graebe, S. F.; Salgado, M. E. (2001). Control system design, Prentice Hall, Upper Saddle Rive, New Jersey

[3] Dutton, K.; Thompson, S.; Barraclough, B. (1997). The art of control engineering, Addision Wesley Longman, Pearson Education, Essex

[4] ALSTOM Power, Ltd. (1998) Materials of the company from the project, OTROKOVICE (in Czech)

[5] Kadrnozka, J.; Ochrana, L. (2001). Combined heat and power production, CERM, Brno (in Czech)

[6] Kadrnožka, J. (2004). Thermal-turbine and turbo-compressors, CERM, Brno (in Czech)

[7] Åström, K.; Hägglund, T. (1995). PID Controllers: Theory, Design, and Tuning, $2^{\text {nd }}$ edition, Instrument Society of America, Research Triangle Park, North Carolina

[8] Viteckova, M. (1998). Adjustment of Controllers by the method of Dynamics Inversion, Lecture notes of VŠB-TU Ostrava (in Czech)

[9] Viteckova, M.; Vitecek, A. (2008). Bases of automatic control, $2^{\text {nd }}$ edition, VŠB - Technical University Ostrava, Ostrava (in Czech)

[10] Wagnerova, R.; Minar, M. Control loop synthesis, from http://www.fs.vsb.cz/fakulta/kat/352/ uc_texty/synteza/index.htm, accessed on 2000-08-22

[11] Kiong, K. K.; Guo, W. Q.; Chien, H. C.; Hägglund, T. (1999). Advances in PID control, Springer-Verlag, London

[12] Prokop, R.; Matusu, R.; Prokopova, Z. (2006). Automatic control theory - linear continuous dynamic systems, TBU in Zlin, ISBN 80-7317-369-2, Zlin (in Czech)

[13] Karban, P. (2006). Computing and simulation in program Matlab and Simulink, Computer Press, Brno (in Czech)

[14] Navratil, P., Balate, J. (2007). One of possible approaches to control of multivariable control loop, Proceedings of the $8^{\text {th }}$ International IFAC Symposium on Dynamics and Control of Process Systems, Vol. 1, 207-212 